\title{
Asthma and pregnancy: do they give clues to abnormal antiviral activity?
}

\author{
William W Busse, ${ }^{1}$ James E Gern²
}

Patients with asthma and pregnancy were found as two groups most at risk for severe respiratory outcomes during the H1N1 epidemic in 2008. ${ }^{1-4}$ This association raises the question as to what immune dysfunctions may link these two conditions together and lead to an increased susceptibility for viral respiratory infections, and whether both patient populations may have similar defects in antiviral activity. This important and interesting clinical question is the focus of the paper by Forbes et al ${ }^{5}$ in Thorax.

In both asthma and pregnancy, there is a loss of the normal Th1/Th2 balance. ${ }^{6} 7$ In pregnancy, the shift away from a Th1 expression and towards a Th2 dominance during gestation likely reflects an 'immune necessity' to prevent the mother from having a 'graft versus host' reaction and subsequent loss of the pregnancy. ${ }^{7}$ In asthma, the immune picture is more complex, but the possible contribution of a Th1/Th2 imbalance with the presence of allergic reactions has been the basis of the hygiene hypothesis and target of new treatments. ${ }^{8}$ In broad-based terms, allergic sensitisation represents an overexpression of Th2 cytokines, interleukin (IL)-4, IL-5 and IL-13, with the resulting production of IgE antibodies with a capacity to cause allergic inflammation following allergen activation. In asthma, and pregnancy, a shift towards Th2 dominance also means a possible reduction in Th1 cytokines, such as interferons. The consequence of diminished interferon generation could explain the increased risk for respiratory infections in both asthma and pregnancy.

\footnotetext{
${ }^{1}$ Department of Medicine, University of Wisconsin School of Medicine and Public Health, Madison, Wisconsin, USA; '2Department of Pediatrics, University of Wisconsin School of Medicine and Public Health, Madison, Wisconsin, USA

Correspondence to Professor William W Busse, Medicine, University of Wisconsin Hospital, K4/910 CSC, MC 9988, 600 Highland Avenue, Madison, WI 53792, USA, wwb@medicine.wisc.edu
}

A deficiency in the generation of interferon has been most extensively studied in asthma and has been proposed as a risk factor for respiratory infections, primarily rhinoviruses, which then lead to exacerbations of underlying airway disease..$^{9-11}$ Because pregnancy may also have deficiencies in interferon expression, there exists a similar possibility for a greater frequency, and severity, of respiratory tract infections. As a consequence of these observations, the study by Forbes et $a l^{5}$ is of particular interest to clarify the specificity of altered interferon generation to asthma alone and whether pregnancy may also lead to similar deficiencies. In both situations, this question is of clinical relevance.

Using their Managing Asthma in Pregnancy study group, Forbes, et a $t^{5}$ evaluated in vitro peripheral blood mononuclear cell (PBMC) generation of interferon $\alpha$ (Type I) and $\lambda$ (Type III) to a variety of activators: rhinoviruses (HRV43-major group and RV1B-minor group), a TLR3 agonist, polyinosine-polycytidylic acid (Poly(I:C) and a TLR agonist, imiquimod. By using this panel of activators, the investigators could gain insight into the various pathways of cell stimulation under which deficiencies in interferon production may occur as well as determining whether asthma and pregnancy share a common abnormality.

Based upon the clinical experiences with $\mathrm{H} 1 \mathrm{N1}$, it is not surprising that pregnancy in healthy controls was associated with decreased PBMC generation of interferon $\alpha$ and $\lambda$ to a respiratory virus, which in this study included major and minor groups of rhinovirus. Also not surprisingly was the variability to which these responses were altered in individual patients. Re-testing normal controls in the postpartum period found the generation of interferons to rhinoviruses gradually returning to normal suggesting that this defect was not 'permanent' but related to the pregnancy rather than an underlying abnormality in the subjects tested. Similarly, when asthma patients were preg- nant, there was an associated decrease in interferon $\lambda$ generation when compared with healthy controls. In apparent contrast to other observations, ${ }^{12}$ the interferon response in patients with stable asthma, at least with PBMC, was not different from healthy controls. Finally, in asthmatic women who were pregnant and recovering from an exacerbation, the generation of interferons was the lowest of all the groups tested. Is this information helpful or does it further confuse an already complex pattern that is emerging from a variety of laboratories?

There has been considerable interest and excitement that a key feature of some asthma patients is a reduced generation of interferon to respiratory viruses. This abnormality has been observed with samples of blood mononuclear cells, ${ }^{12-14}$ dendritic cells ${ }^{15}$ and airway epithelial cells, although the latter is not a consistent finding in all studies. ${ }^{10} 11$ 16-18 These findings raise additional questions about the mechanisms that impair antiviral responses in asthma. There is evidence that the quality of antiviral responses can be influenced by genetics, or is imprinted in early life as a consequence of viral lower respiratory tract infections, microbial exposures or acquisition of allergies. ${ }^{19-21}$ The study by Forbes et $a l^{5}$ indicates that deficiencies in interferon generation to rhinoviruses are not limited to asthma, but can also occur in other conditions like pregnancy, and in pregnancy, at least, these defects are transient.

Notably, mechanisms for transient reductions in interferon generation have been described. For example, cross-linking IgE receptors on the surface of plasmacytoid dendritic cells impair subsequent interferon responses to viral infection in vitro. ${ }^{15}$ This observation suggests that in sensitised individuals, exposure to an aeroallergen could temporarily interfere with antiviral responses. This theory is consistent with clinical observations that sensitisation and exposure is a strong risk factor for viral wheeze. ${ }^{9}$ It will be of great interest to identify the corresponding, and perhaps novel mechanisms, for reduced interferon responses in pregnancy.

How can the differences between the various studies on defects in interferons be resolved in light of the data by Forbes et al ? ${ }^{5}$ In studies from Sebastian Johnston's laboratory, defects in interferon generation have been noted primarily with epithelial cells. ${ }^{10} 11$ As epithelial cells are the principal target for rhinovirus infection, the observations by Johnston's group are highly relevant as a risk factor 
for respiratory virus infections and subsequent asthma exacerbations. The findings of Johnston and colleagues also suggest that defects in epithelial cells may arise as the result of locally generated airway inflammatory mediators in asthma. The effect of locally generated inflammatory mediators on bronchial cells raises the possibility that antiviral abnormalities in asthma are localised to affected tissues, e.g. epithelial cells, and most readily detected in these samples. In contrast, Forbes et $a l^{5}$ used PBMC to assess the effect of pregnancy on interferon generation. Because pregnancy has systemic effects, it is possible that their described abnormalities with PBMC would be found in circulating cells exposed to immune modifying factors associated with pregnancy. Further work will be necessary to resolve this issue but we suggest that there is a compartmentalisation of these antiviral, interferon-associated defects, which is dependent on the nature of the underlying condition, that is, local (asthma) versus systemic (pregnancy).

Although the observations by Forbes et $a l^{5}$ extend information of diminished interferon generation and risk factors for a severe viral respiratory infection, there are inherent limitations to this current study. The number of subjects studied was small (approximately 10 per group), which prevents comprehensive and definitive comparisons of interferon responses in pregnancy and phenotypic features of asthma. Also, the greatest reduction in interferon generation was found in a very limited number $(n=4)$ of pregnant asthma patients who were evaluated after they had experienced an exacerbation. Whether the presence of this abnormality in interferon generation is a cause or consequence of the combination of asthma and the exacerbation is not known. Finally, the authors demonstrated that interferon responses to rhinovirus and TLR7 were impaired in asthma. There are several other pathways for rhinoviruses to induce interferon, and additional studies are needed to determine whether these observations are causally related.

Besides the possibility that the development of transient interferon deficiency may explain an enhanced risk for exacerbation during pregnancy, the importance of these observations is to tantalise the research community into seeking similarities between pregnancy and asthma. Perhaps pregnancy is a model system to more fully understand what can happen during a limited time frame to suppress antiviral interferon production, and with this knowledge we can possibly gain insight into the mechanisms of the altered interferon response as well as new therapeutic approaches to prevent exacerbations of asthma from respiratory infections.

Competing interests None.

Provenance and peer review Commissioned; internally peer reviewed.

Published Online First 8 October 2012

Thorax 2012;67:189-190

doi:10.1136/thoraxjnl-2011-201007

\section{REFERENCES}

1. CDC Estimates of 2009 H1N1 Influenza Cases, Hospitalizations and Deaths in the United States, April 2009-March 13, 2010. Atlanta: Centers for Disease Control and Prevention, 2010.

2. Jain S, Kamimoto L, Bramley AM, et al; 2009 Pandemic Influenza A (H1N1) Virus Hospitalizations Investigation Team. Hospitalized patients with 2009 H1N1 influenza in the United States, April-June 2009. N Engl J Med 2009;361:1935-44.

3. 2009 H1N1 Flu: Underlying Health Conditions Among Hospitalized Adults and Children. Centers for Disease Control and Prevention, 2010. http://www.cdc.gov/ H1N1flu/eip underlying conditions.htm laccessed 24 Aug 2011)

4. Jamieson DJ, Honein MA, Rasmussen SA, et al; Novel Influenza A (H1N1) Pregnancy Working Group. H1N1 2009 influenza virus infection during pregnancy in the USA. Lancet 2009;374:451-8.

5. Forbes R, Gibson P, Wark P, et al. Impaired type I and type III interferon response to rhinovirus infection during pregnancy and asthma. Thorax 2012;67:209-314.
6. Strachan DP. Hay fever, hygiene, and household size. BMJ 1989:299:1259-60.

7. Aris A, Lambert F, Bessette $P$, et al. Maternal circulating interferon-gamma and interleukin-6 as biomarkers of Th1/Th2 immune status throughout pregnancy. J Obstet Gynaecol Res 2008;34:7-11.

8. Busse WW, Lemanske RF Jr. Asthma. N Eng/ J Med 2001:344:350-62.

9. Busse WW, Lemanske RF Jr, Gern JE. Role of vira respiratory infections in asthma and asthma exacerbations. Lancet 2010;376:826-34.

10. Wark PA, Johnston SL, Bucchieri F, et al. Asthmatic bronchial epithelial cells have a deficient innate immune response to infection with rhinovirus. J Exp Med 2005;201:937-47.

11. Contoli M, Message SD, Laza-Stanca V, et al. Role of deficient type III interferon-lambda production in asthma exacerbations. Nat Med 2006;12:1023-6.

12. Papadopoulos NG, Stanciu LA, Papi A, et al. A defective type 1 response to rhinovirus in atopic asthma. Thorax 2002;57:328-32.

13. Parry DE, Busse WW, Sukow KA, et al. Rhinovirusinduced PBMC responses and outcome of experimental infection in allergic subjects. J Allergy Clin Immunol 2000;105:692-8.

14. Brooks GD, Buchta KA, Swenson CA, et al. Rhinovirus-induced interferon-gamma and airway responsiveness in asthma. Am J Respir Crit Care Med 2003:168:1091-4.

15. Gill MA, Bajwa G, George TA, et al. Counterregulation between the FcepsilonRI pathway and antiviral responses in human plasmacytoid dendritic cells. J Immunol 2010:184:5999-6006.

16. Lopez-Souza N, Favoreto $S$, Wong $H$, et al. In vitro susceptibility to rhinovirus infection is greater for bronchial than for nasal airway epithelial cells in human subjects. J Allergy Clin Immunol 2009; 123:1384-90.

17. Rosenthal LA, Avila PC, Heymann PW, et al; Infections and Asthma Committee, Environmental and Occupational Respiratory Diseases Interest Section, American Academy of Allergy, Asthma \& Immunology. Viral respiratory tract infections and asthma: the course ahead. J Allergy Clin Immunol 2010;125:1212-17.

18. Bochkov YA, Hanson KM, Keles S, et al. Rhinovirusinduced modulation of gene expression in bronchial epithelial cells from subjects with asthma. Mucosal Immunol 2010;3:69-80.

19. Sly PD, Boner AL, Bjorksten B, et al. Early identification of atopy in the prediction of persistent asthma in children. Lancet 2008;372:1100-6

20. Benoit LA, Holtzman MJ. New immune pathways from chronic post-viral lung disease. Ann N Y Acad Sci 2010;1183:195-210.

21. von Mutius E, Vercelli D. Farm living: effects on childhood asthma and allergy. Nat Rev Immunol 2010;10:861-8. 